Article

\title{
A New Iterative Predictor-Corrector Algorithm for Solving a System of Nuclear Magnetic Resonance Flow Equations of Fractional Order
}

\author{
Mariam Sultana ${ }^{1, *} \mathbb{\infty}$, Uroosa Arshad ${ }^{1}\left(\mathbb{D}\right.$, Muhammad Khalid $^{1}$, Ali Akgül ${ }^{2} \oplus$, Wedad Albalawi ${ }^{3}$ \\ and Heba Y. Zahran 4,5
}

check for

updates

Citation: Sultana, M.; Arshad, U.;

Khalid, M.; Akgül, A.; Albalawi, W.;

Zahran, H.Y. A New Iterative

Predictor-Corrector Algorithm for

Solving a System of Nuclear

Magnetic Resonance Flow Equations

of Fractional Order. Fractal Fract.

2022, 6, 91. https://doi.org/

10.3390 / fractalfract6020091

Academic Editor: Manuel

Duarte Ortigueira

Received: 5 December 2021

Accepted: 30 January 2022

Published: 6 February 2022

Publisher's Note: MDPI stays neutral with regard to jurisdictional claims in published maps and institutional affiliations.

Copyright: () 2022 by the authors Licensee MDPI, Basel, Switzerland. This article is an open access article distributed under the terms and conditions of the Creative Commons Attribution (CC BY) license (https:// creativecommons.org/licenses/by/ $4.0 /)$.
1 Department of Mathematical Sciences, Sciences and Technology, Gulshan-e-Iqbal Campus, Federal Urdu University of Arts, University Road, Karachi 75300, Pakistan; uroosaarshad_24@yahoo.com (U.A.); khalidsiddiqui@fuuast.edu.pk (M.K.)

2 Art and Science Faculty, Department of Mathematics, Siirt University, Siirt 56100, Turkey; aliakgul00727@gmail.com

3 Department of Mathematical Sciences, College of Science, Princess Nourah bint Abdulrahman University, P.O. Box 84428, Riyadh 11671, Saudi Arabia; wsalbalawi@pnu.edu.sa

4 Laboratory of Nano-Smart Materials for Science and Technology (LNSMST), Department of Physics, Faculty of Science, King Khalid University, P.O. Box 9004, Abha 61413, Saudi Arabia; heldemardash@kku.edu.sa

5 Nanoscience Laboratory for Environmental, Biomedical Applications (NLEBA), Metallurgical Lab. 1, Department of Physics, Faculty of Education, Ain Shams University, Roxy, Cairo 11757, Egypt

* Correspondence: marium.sultana@fuuast.edu.pk

Abstract: Nuclear magnetic resonance flow equations, also known as the Bloch system, are said to be at the heart of both magnetic resonance imaging (MRI) and nuclear magnetic resonance (NMR) spectroscopy. The main aim of this research was to solve fractional nuclear magnetic resonance flow equations (FNMRFEs) through a numerical approach that is very easy to handle. We present a New Iterative Predictor-Corrector Algorithm (NIPCA) based on the New Iterative Algorithm and Predictor-Corrector Algorithm to solve nonlinear nuclear magnetic resonance flow equations of fractional order involving Caputo derivatives. Graphical representation of the solutions with detailed error analysis shows the higher accuracy of the new technique. This New Iterative Predictor-Corrector Algorithm requires less computational time than previously published numerical methods. The results achieved in this article indicate that the algorithm is fit to use for other chaotic systems of fractional differential equations.

Keywords: fractional calculus; fractional nuclear magnetic resonance flow equations; magnetic resonance imaging; New Iterative Algorithm; nuclear magnetic resonance; Predictor-Corrector Algorithm

\section{Introduction}

Nuclear magnetic resonance provides the physical basis for a vast selection of methods that are usually used to investigate the dynamics of the structure of cells, tissues, etc., up to the extent of the entire body [1]. For example, chemists have recently studied biomolecules and their structural analysis using magnetic resonance spectroscopy (MRS), and MRI is a vital instrument in the radiology departments of hospitals. MRI helps construct a model of the soft tissue structures of the human spine and brain [2] with a resolution of submillimeter. By comparison, MRS helps in identifying individual bimolecular structural configurations with a resolution up to sub-nanometer. This provides a tremendously broad range of scales, providing the physician with a safe means to identify diseases and their stages, such as cancer, and provides chemists with a highly efficient instrument for understanding molecular synthesis. For more details, see [3-7].

Similarly, for discovering the molecular basis that underlies abnormal cell growth, spectroscopic and imaging information provides the necessary technological tools, in addition to helping in monitoring a unique tumor's response to drugs or radiological treatments. 
The typical means of defining NMR (i.e., "the phenomena that make up the inner workings of $\left.M R I^{\prime \prime}\right)$ in vector form is presented with the help of the Bloch equation. This equation corresponding to a uniform sample may be expressed as [8,9]:

$$
\frac{d \vec{M}}{d t}=\Gamma(\vec{M} \times \vec{B})-\frac{M_{z}-M_{o}}{T_{1}^{\prime}} \hat{i}_{z}-\frac{M_{x} \hat{i}_{x}+M_{y} \hat{i}_{y}}{T_{2}^{\prime}}
$$

where $\vec{M}\left(M_{x}, M_{y}, M_{z}\right)$ is the time-varying system magnetization; $M_{0}$ represents the equilibrium magnetization; $\vec{B}\left(B_{x}, B_{y}, B_{z}\right)$ is the applied radio frequency $B_{x}$, gradient $B_{y}$, and static magnetic fields $B_{z} ; \Gamma$ is the gyro-magnetic ratio; and $T_{1}^{\prime}$ shows the spin-lattice relaxation time, giving the characterization of the rate at which the longitudinal $M_{z}$ component of the magnetization vector recovers. It has the property that it changes exponentially towards its thermodynamic equilibrium. $T_{2}^{\prime}$ is the spin-spin relaxation time, which gives the characterization of the signal decay in MRI and NMR, i.e., $T_{2}^{\prime}$ is the rate that corresponds to the exponential decay of the zero towards the transverse component of the magnetization vector, $M_{x y}=M_{x} \hat{i}_{x}+M_{y} \hat{i}_{y}$.

In this paper, we present, for the first time, a new technique for the numerical solution of fractional nuclear magnetic resonance flow equations, in the form of the technique of the New Iterative Predictor-Corrector Algorithm. In Section 2, we discuss nuclear magnetic resonance flow equations of fractional order, including information about the Caputo derivative. Section 3 describes a New Iterative Predictor-Corrector Algorithm specially formulated on nonlinear fractional nuclear magnetic resonance flow. In Section 4, the numerical simulation is presented. In the last section, a conclusive summary of the research is presented.

\section{Application on Fractional Nuclear Magnetic Resonance Flow Equations}

Nuclear magnetic resonance flow equations can be considered as a system of macroscopic equations. These equations estimate nuclear magnetization as a function of time $\vec{M}\left(M_{x}, M_{y}, M_{z}\right)$ with the relaxation times of spin-lattice $T_{1}^{\prime}$ and spin-spin $T_{2}^{\prime}$. Felix Bloch is the pioneer who first introduced these equations in 1946. Later developments in the research of fractional calculus have shown that a system of fractional order differential equations involving Caputo derivatives enables a mathematical description in the following form [10]:

$$
\begin{aligned}
& D_{t}^{\alpha} M_{x}(t)=\omega_{0}^{\prime} M_{y}(t)-\frac{M_{x}(t)}{T_{2}^{\prime}} \\
& D_{t}^{\beta} M_{y}(t)=-\omega_{0}^{\prime} M_{x}(t)-\frac{M_{y}(t)}{T_{2}^{\prime}} \\
& D_{t}^{\eta} M_{z}(t)=\frac{M_{0}-M_{z}(t)}{T_{1}^{\prime}}
\end{aligned}
$$

where $M_{0}$ is the equilibrium magnetization. The Larmor relationship provides the resonant frequency $\omega_{0}^{\prime}$ as: $\omega_{0}^{\prime}=\Gamma B_{0}$. Here, $B_{0}$ is the constant static magnetic field. In the case of the gyro-magnetic ratio $\frac{\Gamma}{2 \pi}=\frac{f_{0}}{B_{0}}=42.57 \mathrm{MHz} / \mathrm{T}$ corresponding to water protons, we have $\omega_{0}^{\prime}=2 \pi f_{0}$.

Moreover, in order to maintain consistency in setting the units measuring the magnetization, $s^{\bar{\alpha}}$ is chosen to express the measurements of $T_{1}^{\prime}, T_{2}^{\prime}$, and $\omega_{0}^{\prime}$. Symbols $\alpha$, $\beta$, and $\eta$ denote Caputo derivative orders, with $\bar{\alpha}=(\alpha, \beta, \eta)$ denoting the order of the total system.

The Caputo fractional derivative is a better substitute than the Riemann-Liouville type for computing the fractional derivative because it does not require fractional order initial conditions. With $\alpha$ denoting the fractional order, we can express the fractional order derivative of a function $f$ in the Caputo sense as:

$$
{ }_{b}^{C} D_{t}^{\alpha} f(t)=\frac{1}{\Gamma(p-\alpha)} \int_{b}^{t} \frac{f^{(p)}(\eta)}{(t-\eta)^{\alpha+1-\eta}} d \eta, \quad(p-1<\alpha<p)
$$


The Caputo fractional derivative of the $\alpha$ th order can be simplified as $D^{\alpha}$ if $b=0$.

For $\alpha=1$, the Caputo sense derivative becomes $D^{\alpha} f(t)=\frac{d f(t)}{d t}$. Some important properties of Caputo fractional derivative are given below:

(a). $D_{t}^{\alpha} t^{\Gamma}=\frac{\Gamma(1+\Gamma)}{\Gamma(1+\Gamma-\alpha)} t^{\Gamma-\alpha} ; \Gamma>0$.

(b). $D_{t}^{\alpha}(\delta f(t)+\tau g(t))=\delta D_{t}^{\alpha} f(t)+\tau D_{t}^{\alpha} g(t)$, where $\delta$ and $\tau$ are constant.

(c). $D_{t}^{\alpha} D_{t}^{q} f(t)=D_{t}^{\alpha+q} f(t) \neq D_{t}^{q} D_{t}^{\alpha} f(t), \alpha \in R, q \in N$.

(d). $D_{t}^{\alpha} c=0$.

where (b) is known as the linearity property and (c) is known as the non-commutative property of the Caputo fractional derivative. For more detail, see [11].

\section{New Iterative Predictor-Corrector Algorithm (NIPCA)}

A variety of problems in biology, physics, engineering, and chemistry give rise to relations that are expressed in the form of nonlinear functional equations. Therefore, consider the equation:

$$
u=g+\aleph(u)
$$

where $\aleph$ is a nonlinear operator and $g$ is a known function. There are various techniques to solve this nonlinear functional equation, such as the Adomian Decomposition Method [12,13], the Homotopy Perturbation Method [14], the New Iterative Method [15-19], the New Perturbation Iteration Transform Method [20], and the Perturbation Iteration Algorithm [21]. In this method, the nonlinear operator $\aleph$ can be decomposed as:

$$
\aleph(u)=\aleph\left(u_{0}\right)+\left[\aleph\left(u_{0}+u_{1}\right)-\aleph\left(u_{o}\right)\right]+\ldots
$$

Suppose $\mathrm{H}_{o}=\aleph\left(u_{o}\right)$ and $\mathrm{H}_{i}=\aleph\left(\sum_{m=0}^{i} u_{m}\right)-\aleph\left(\sum_{m=0}^{i-1} u_{m}\right)$ for $i=1,2,3, \ldots$

Note that $\aleph(u)=\sum_{i=0}^{\infty} \mathrm{H}_{i}$. Put $u_{o}=g$ and $u_{m}=\mathrm{H}_{m-1}$ for $m=1,2,3, \ldots$

Observe that:

$$
\begin{aligned}
& u=\sum_{m=0}^{\infty} u_{m} \\
& u=g+\sum_{m=1}^{\infty} \mathrm{H}_{m-1} \\
& u=g+\aleph\left(u_{0}\right)+\left[\aleph\left(u_{0}+u_{1}\right)-\aleph\left(u_{0}\right)\right]+\cdots \\
& u=g+\aleph(u) .
\end{aligned}
$$

Hence, $u$ satisfies the functional Equation (3). We can derive a numerical algorithm of the fractional Bloch system. A mathematical formulation of this system can be expressed as:

$$
\begin{aligned}
& D_{t}^{\alpha} M_{x}(t)=\omega_{0}^{\prime} M_{y}(t)-\frac{M_{x}(t)}{T_{2}^{\prime}}, \quad \alpha>0 \\
& D_{t}^{\beta} M_{y}(t)=-\omega_{0}^{\prime} M_{x}(t)-\frac{M_{y}(t)}{T_{2}^{\prime}}, \quad \beta>0 \\
& D_{t}^{\eta} M_{z}(t)=\frac{M_{0}-M_{z}(t)}{T_{1}^{\prime}}, \quad \eta>0
\end{aligned}
$$

This system can be rewritten as:

$$
\begin{aligned}
& D_{t}^{\alpha} M_{x}(t)=q_{1}\left(t, M_{x}(t)\right) \\
& D_{t}^{\beta} M_{y}(t)=q_{2}\left(t, M_{y}(t)\right) \\
& D_{t}^{\eta} M_{z}(t)=q_{3}\left(t, M_{z}(t)\right)
\end{aligned}
$$


with initial conditions $M_{x}(0)=0, M_{y}(0)=100, M_{z}(0)=0$. By applying the trapezoidal quadrature formula to the fractional Bloch system, we obtain:

$$
\begin{array}{r}
M_{x}\left(t_{m+1}\right)=M_{x}(0)+\frac{h^{\alpha}}{\Gamma(\alpha+2)} a_{10, m+1} \mathscr{q}_{1}\left(t_{m}, M_{x}\left(t_{m}\right)\right) \\
+\frac{h^{\alpha}}{\Gamma(\alpha+2)} \sum_{j=1}^{m} a_{1 j, m+1} \mathscr{q}_{1}\left(t_{j}, M_{x}\left(t_{j}\right)\right) \\
M_{y}\left(t_{m+1}\right)=M_{y}(0)+\frac{h^{\beta}}{\Gamma(\beta+2)} a_{20, m+1} \mathscr{q}_{2}\left(t_{m}, M_{y}\left(t_{m}\right)\right) \\
+\frac{h^{\beta}}{\Gamma(\beta+2)} \sum_{j=1}^{m} a_{2 j, m+1} \mathscr{q}_{2}\left(t_{j}, M_{y}\left(t_{j}\right)\right) \\
M_{z}\left(t_{m+1}\right)=M_{z}(0)+\frac{h^{\eta}}{\Gamma(\eta+2)} a_{30, m+1} \mathscr{q}_{3}\left(t_{m}, M_{z}\left(t_{m}\right)\right) \\
+\frac{h^{\eta}}{\Gamma(\eta+2)} \sum_{j=1}^{m} a_{3 j, m+1} \mathscr{q}_{3}\left(t_{j}, M_{z}\left(t_{j}\right)\right)
\end{array}
$$

where:

$$
a_{j, m+1}=\left\{\begin{array}{clc}
m^{\alpha+1}-(m-\alpha)(m+1)^{\alpha} & \text { if } & j=0 \\
(m-j+2)^{\alpha+1}+(m-j)^{\alpha+1}-2(m-j+1)^{\alpha+1} & \text { if } & 1 \leq j \leq m \\
1 & \text { if } & j=m+1 .
\end{array}\right.
$$

From the algorithm of New Iterative Method [22], we have:

$$
\begin{aligned}
& M_{x, 0}\left(t_{m+1}\right)=M_{x}(0)+\frac{h^{\alpha}}{\Gamma(\alpha+2)} a_{10, m+1} \mathscr{q}_{1}\left(t_{m}, M_{x}\left(t_{m}\right)\right) \\
& M_{y, 0}\left(t_{m+1}\right)=M_{y}(0)+\frac{h^{\beta}}{\Gamma(\beta+2)} a_{20, m+1} q_{2}\left(t_{m}, M_{y}\left(t_{m}\right)\right) \\
& M_{z, 0}\left(t_{m+1}\right)=M_{z}(0)+\frac{h^{\eta}}{\Gamma(\eta+2)} a_{30, m+1} q_{3}\left(t_{m}, M_{z}\left(t_{m}\right)\right)
\end{aligned}
$$

Moreover, we must note that:

$$
\begin{aligned}
& M_{x, 1}\left(t_{m+1}\right)=\aleph_{1}\left(M_{x, 0}, M_{y, 0}, M_{z, 0}\right) \\
& M_{y, 1}\left(t_{m+1}\right)=\aleph_{2}\left(M_{x, 0}, M_{y, 0}, M_{z, 0}\right) \\
& M_{z, 1}\left(t_{m+1}\right)=\aleph_{3}\left(M_{x, 0}, M_{y, 0}, M_{z, 0}\right)
\end{aligned}
$$

where:

$$
\aleph_{1}\left[M_{x}\left(t_{m+1}\right)\right]=\frac{h^{\alpha}}{\Gamma(\alpha+2)} g_{1}\left(t_{m+1}, M_{x}\left(t_{m+1}\right)\right)
$$

At the $k$ th iteration, $k=2,3, \ldots$

$$
\begin{aligned}
& M_{x, k}=\aleph_{1}\left(\sum_{i=0}^{k-1} M_{x, i}, \sum_{i=0}^{k-1} M_{y, i}, \sum_{i=0}^{k-1} M_{z, i}\right)-\aleph_{1}\left(\sum_{i=0}^{k-2} M_{x, i}, \sum_{i=0}^{k-2} M_{y, i}, \sum_{i=0}^{k-2} M_{z, i}\right) \\
& M_{y, k}=\aleph_{2}\left(\sum_{i=0}^{k-1} M_{x, i}, \sum_{i=0}^{k-1} M_{y, i}, \sum_{i=0}^{k-1} M_{z, i}\right)-\aleph_{2}\left(\sum_{i=0}^{k-2} M_{x, i}, \sum_{i=0}^{k-2} M_{y, i}, \sum_{i=0}^{k-2} M_{z, i}\right) \\
& M_{z, k}=\aleph_{3}\left(\sum_{i=0}^{k-1} M_{x, i}, \sum_{i=0}^{k-1} M_{y, i}, \sum_{i=0}^{k-1} M_{z, i}\right)-\aleph_{3}\left(\sum_{i=0}^{k-2} M_{x, i}, \sum_{i=0}^{k-2} M_{y, i}, \sum_{i=0}^{k-2} M_{z, i}\right)
\end{aligned}
$$


Note that $M_{i}=\sum_{j=0}^{\infty} M_{i, j}, i=x, y, z$ and $M_{i}=\left(M_{x}, M_{y}, M_{z}\right)$ comprises a solution of the given system of Bloch equations of fractional order. Using the New Iteration Algorithm together with the Predictor-Corrector Algorithm, the following approximations were calculated:

$$
\begin{aligned}
& y_{1, m+1}^{p}=M_{x}(0)+\frac{h^{\alpha}}{\Gamma(\alpha+2)} \sum_{j=0}^{m} a_{1 j, m+1} \mathscr{Z}_{1}\left(t_{j}, M_{x}\left(t_{j}\right)\right) \\
& y_{2, m+1}^{p}=M_{y}(0)+\frac{h^{\beta}}{\Gamma(\beta+2)} \sum_{j=0}^{m} a_{2 j, m+1} \mathscr{q}_{2}\left(t_{j}, M_{y}\left(t_{j}\right)\right) \\
& y_{3, m+1}^{p}=M_{z}(0)+\frac{h^{\eta}}{\Gamma(\eta+2)} \sum_{j=0}^{m} a_{3 j, m+1} \mathscr{q}_{3}\left(t_{j}, M_{z}\left(t_{j}\right)\right)
\end{aligned}
$$

and:

$$
\begin{aligned}
z_{1, m+1}^{p} & =\frac{h^{\alpha}}{\Gamma(\alpha+2)} g_{1}\left(t_{m+1}, y_{1, m+1}^{p}\right) \\
z_{2, m+1}^{p} & =\frac{h^{\beta}}{\Gamma(\beta+2)} g_{2}\left(t_{m+1}, y_{2, m+1}^{p}\right) \\
z_{3, m+1}^{p} & =\frac{h^{\eta}}{\Gamma(\eta+2)} g_{3}\left(t_{m+1}, y_{3, m+1}^{p}\right)
\end{aligned}
$$

Similarly:

$$
\begin{aligned}
& x_{1, m+1}^{c}=y_{1, m+1}^{p}+\frac{h^{\alpha}}{\Gamma(\alpha+2)} g_{1}\left(t_{m+1}, y_{1, m+1}^{p}+z_{1, m+1}^{p}\right) \\
& x_{2, m+1}^{c}=y_{2, m+1}^{p}+\frac{h^{\beta}}{\Gamma(\beta+2)} g_{2}\left(t_{m+1}, y_{2, m+1}^{p}+z_{2, m+1}^{p}\right) \\
& x_{3, m+1}^{c}=y_{3, m+1}^{p}+\frac{h^{\eta}}{\Gamma(\eta+2)} g_{3}\left(t_{m+1}, y_{3, m+1}^{p}+z_{3, m+1}^{p}\right)
\end{aligned}
$$

Here, $y_{1, m+1}^{p}, y_{2, m+1}^{p}, y_{3, m+1}^{p}, z_{1, m+1}^{p}, z_{2, m+1}^{p}$ and $z_{3, m+1}^{p}$ are the predictor terms and $x_{1, m+1}^{c}, x_{2, m+1}^{c}$ and $x_{3, m+1}^{c}$ are the corrector terms. Here, $M_{x}, M_{y}$ and $M_{z}$ denote the approximate values of the solutions of the FNMRFEs at $t=t_{j}$. With the help of the abovementioned three steps of the New Iterative Predictor-Corrector Algorithm (NIPCA), we solve and discuss nonlinear fractional nuclear magnetic resonance flow equations in the following section.

\section{Results and Discussion}

It is recognized that systems of fractional differential equations powerfully depend upon the initial conditions; therefore, fractional derivatives should be chosen as the most suitable way to handle the initial conditions of physical problems. The system's initial state in NMR is rendered precise by the magnetization components; hence, these must be identified.

A numerical solution for the nonlinear FNMRF system can be derived with the help of NIPCA. A numerical method has the approximate accuracy of a high order, and is a good match with the analytical solution [23]. The starting point or initial condition coefficient is $\left(M_{x}(0), M_{y}(0), M_{z}(0)\right)$.

The coefficients $a_{j, m+1}$ are designed to provide the relation in Equation (5). In this portion, all simulations are performed for different height steps $h$ without the short memory principle. We also calculate the simulation time $T_{\text {sim }}$ with $l=\frac{T_{\text {sim }}}{h}$ and $t_{m}=m h, m=0,1,2, \ldots, l \in Z^{+}$ for every numerical simulation.

The approximate solution of the FNMRF system of equations is illustrated in Figure 1 for $T_{\text {sim }}=0.1 \mathrm{~s}$. In Figure $1 \mathrm{a}, \mathrm{b}$ we examine a limit cycle and plot a spiral, respectively. For $\alpha=\beta=\eta=1.03165$, we obtain the border of critical stability. 


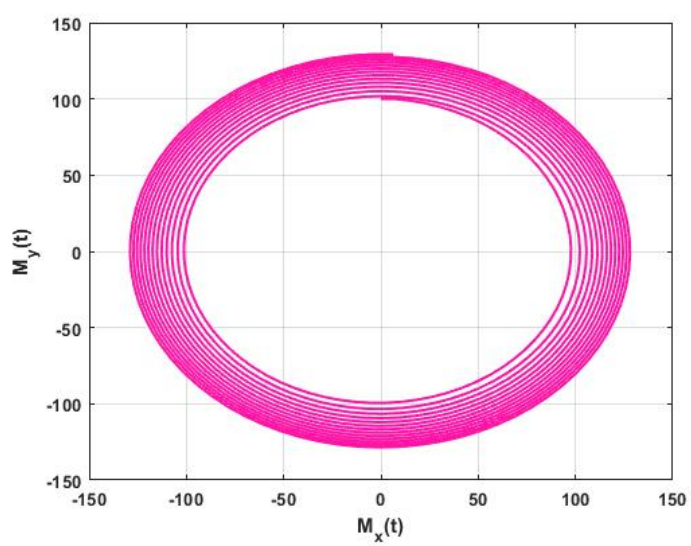

(a)

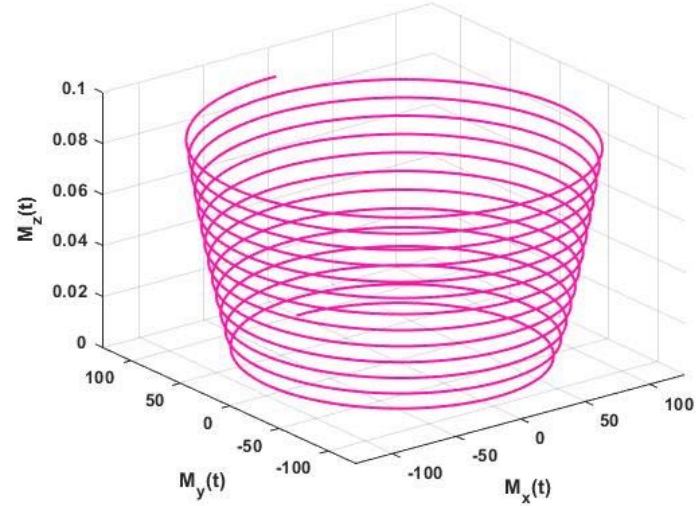

(b)

Figure 1. Approximate solution (a) 2D Plot and (b) 3D Plot of the FNMRF system for $\alpha=\beta=\eta=1.03165$, $T_{2}^{\prime}=20(\mathrm{~ms})^{\bar{\alpha}}, f_{0}=160 \mathrm{~Hz}, T_{1}^{\prime}=1(\mathrm{~s})^{\bar{\alpha}}$ and initial condition $\left(M_{x}(0), M_{y}(0), M_{z}(0)\right)=(0,100,0)$ for $T_{\text {sim }}=0.1 \mathrm{~s}$.

The approximate solution of the FNMRF system of equations is illustrated in Figure 2 for $T_{\text {sim }}=0.2 \mathrm{~s}$ with parameters $\alpha=\beta=\eta=1.03165, T_{2}^{\prime}=20(\mathrm{~ms})^{\alpha}, f_{0}=160 \mathrm{~Hz}, T_{1}^{\prime}=1(\mathrm{~s})^{\alpha}$ with initial condition $\left(M_{x}(0), M_{y}(0), M_{z}(0)\right)=(0,100,0)$ for $T_{s i m}=0.2 \mathrm{~s}$.

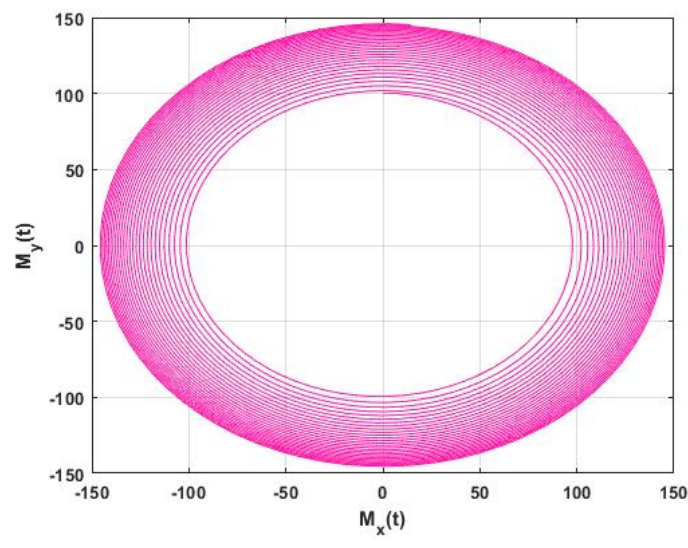

(a)

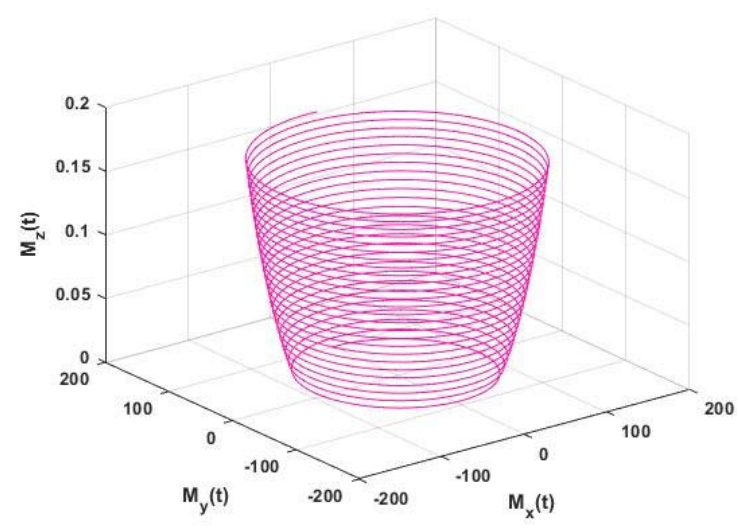

(b)

Figure 2. Approximate solution (a) 2D Plot and (b) 3D Plot of the FNMRF system for $\alpha=\beta=\eta=$ 1.03165 and initial condition $\left(M_{x}(0), M_{y}(0), M_{z}(0)\right)=(0,100,0)$ for $T_{\text {sim }}=0.2 \mathrm{~s}$.

Figure $2 \mathrm{a}, \mathrm{b}$ shows a limit cycle and a spiral plot for $T_{\text {sim }}=0.2 \mathrm{~s}$. In this case, where we consider $\alpha=\beta=\eta=1.03163$ in Equation (4), we have the FNMRF system of equations, and the approximate solution is presented in Figure 3 for $T_{\text {sim }}=1 \mathrm{~s}$. The solution of the FNMRF system with parameters $\alpha=\beta=\eta=1.0, T_{2}^{\prime}=20(\mathrm{~ms})^{\bar{\alpha}}, f_{0}=160 \mathrm{~Hz}, T_{1}^{\prime}=1(\mathrm{~s})^{\bar{\alpha}}$, and initial condition $\left(M_{x}(0), M_{y}(0), M_{z}(0)\right)=(0,100,0)$ for $T_{\text {sim }}=0.1 \mathrm{~s}$ is depicted in Figure 4 for $M_{0}=1$ and in Figure 5 for $M_{0}=-1$. When we consider $\alpha=\beta=\eta=0.9$ in Equation (4), we have the FNMRF model, and the approximate solution is shown in Figure 6 with parameters $\alpha=\beta=\eta=0.9, T_{2}^{\prime}=20(\mathrm{~ms})^{\bar{\alpha}}, f_{0}=160 \mathrm{~Hz}, \mathrm{~T}_{1}^{\prime}=1(\mathrm{~s})^{\bar{\alpha}}$ with initial condition $\left(M_{x}(0), M_{y}(0), M_{z}(0)\right)=(0,100,0)$ for $T_{s i m}=0.2 \mathrm{~s}$ When we consider $\alpha=0.8, \beta=1, \eta=0.9, T_{2}^{\prime}=20(\mathrm{~ms})^{\bar{\alpha}}, f_{0}=160 \mathrm{~Hz}, T_{1}^{\prime}=1(\mathrm{~s})^{\bar{\alpha}}$ and initial condition $\left(M_{x}(0), M_{y}(0), M_{z}(0)\right)=(0,100,0)$ in Equation (4), we have the FNMRF system of Equation (5) and the approximate solution obtained by MATHEMATICA for the $T_{\text {sim }}=0.1 \mathrm{~s}$ is represented in Figure 7. 


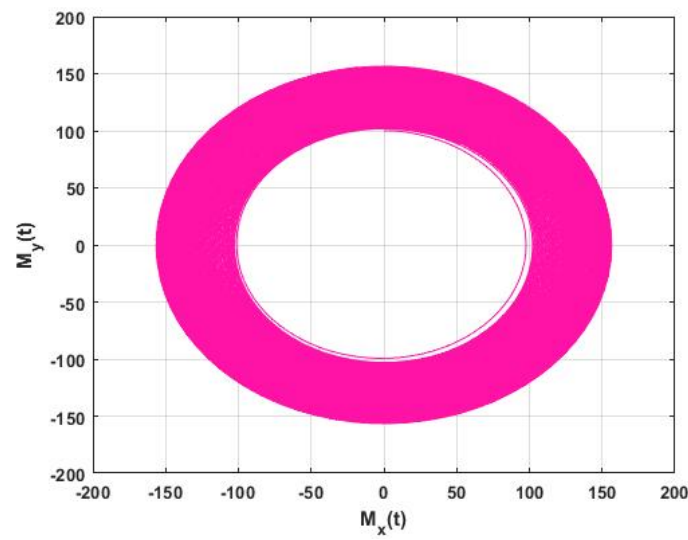

(a)

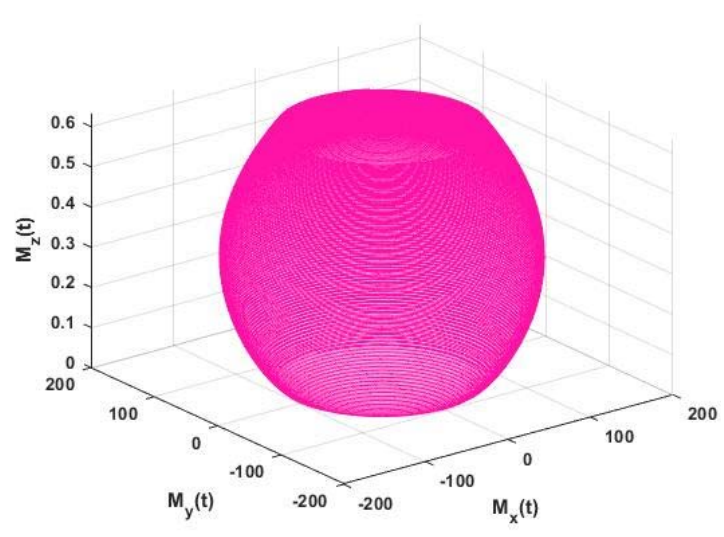

(b)

Figure 3. Approximate solution (a) 2D Plot and (b) 3D Plot of the FNMRF system for $\alpha=\beta=\eta=1.03165$, $T_{2}^{\prime}=20(\mathrm{~ms})^{\bar{\alpha}}, f_{0}=160 \mathrm{~Hz}, T_{1}^{\prime}=1(\mathrm{~s})^{\bar{\alpha}}$ and initial condition $\left(M_{x}(0), M_{y}(0), M_{z}(0)\right)=(0,100,0)$ for $T_{\text {sim }}=1 \mathrm{~s}$.

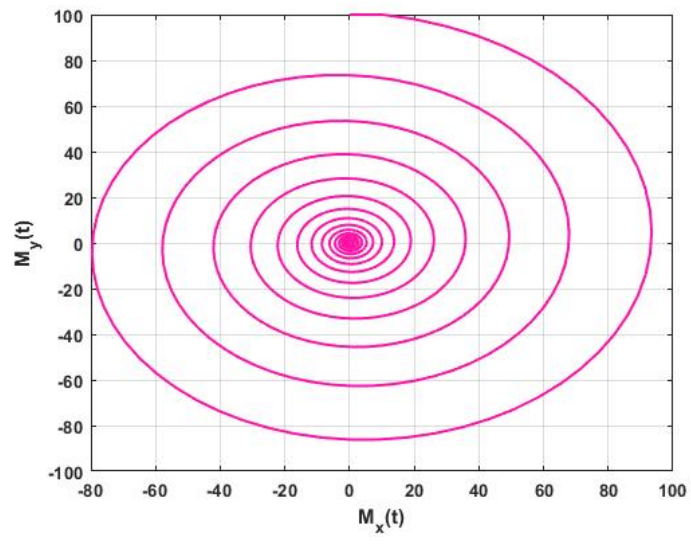

(a)

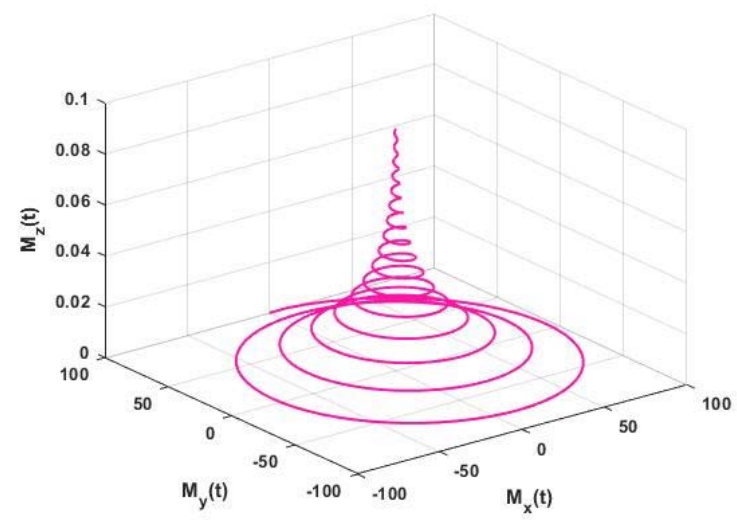

(b)

Figure 4. Approximate solution (a) 2D Plot and (b) 3D Plot of the FNMRF system for $\alpha=\beta=\eta=1.0$, $M_{0}=1$ and initial condition $\left(M_{x}(0), M_{y}(0), M_{z}(0)\right)=(0,100,0)$ for $T_{\text {sim }}=0.1 \mathrm{~s}$.

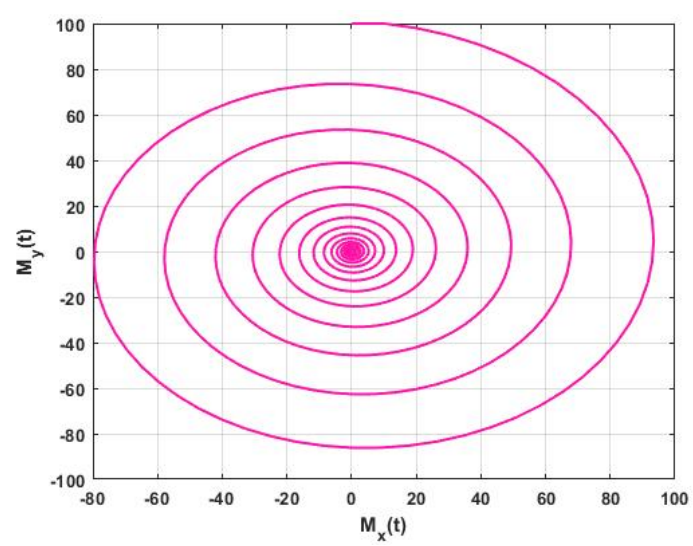

(a)

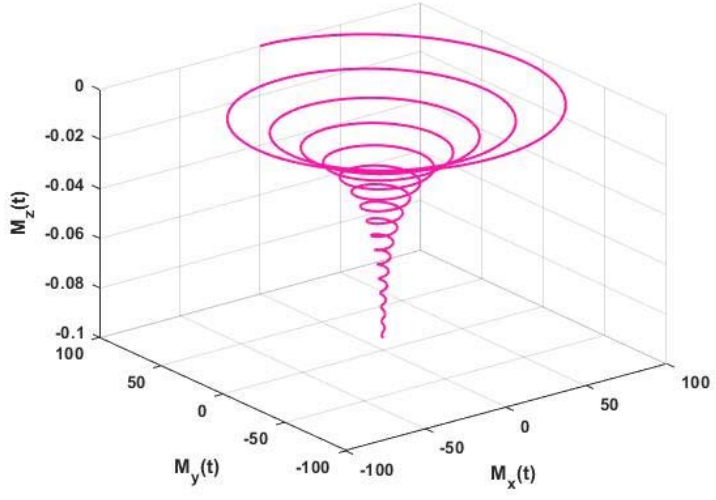

(b)

Figure 5. Approximate solution (a) 2D Plot and (b) 3D Plot of the FNMRF system for $\alpha=\beta=\eta=1.0$, $M_{0}=-1$ and initial condition $\left(M_{x}(0), M_{y}(0), M_{z}(0)\right)=(0,100,0)$ for $T_{\text {sim }}=0.1 \mathrm{~s}$. 


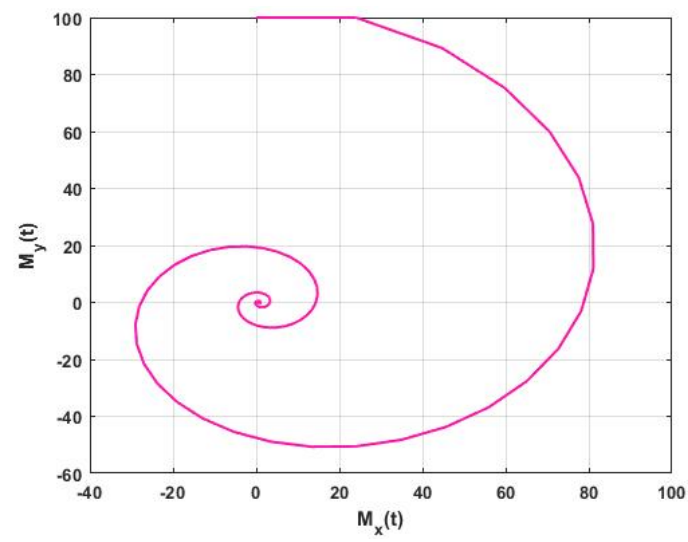

(a)

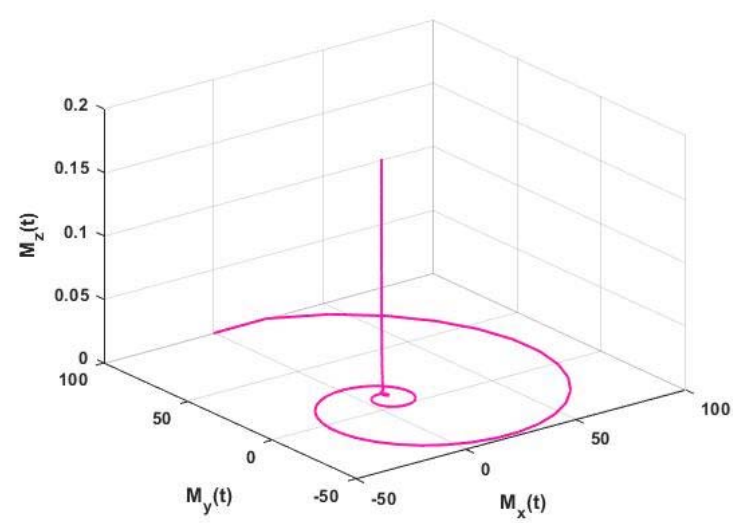

(b)

Figure 6. Approximate solution (a) 2D Plot and (b) 3D Plot of the FNMRF system for $\alpha=\beta=\eta=0.9$ and initial condition $\left(M_{x}(0), M_{y}(0), M_{z}(0)\right)=(0,100,0)$ for $T_{\text {sim }}=0.2 \mathrm{~s}$.

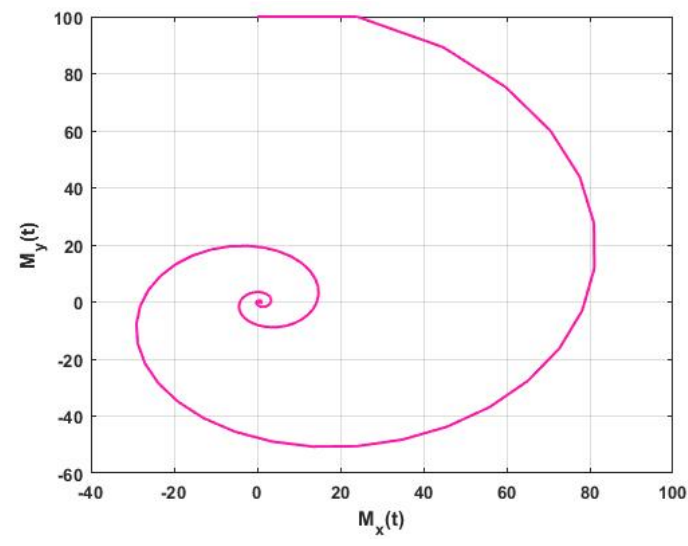

(a)

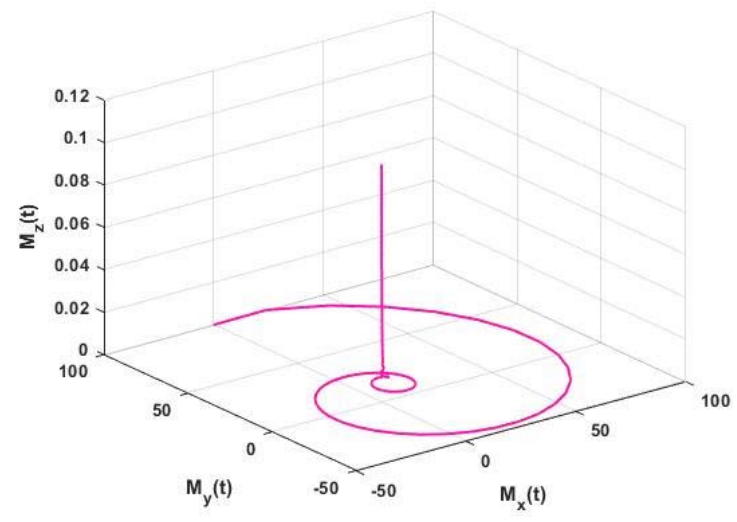

(b)

Figure 7. Approximate solution (a) 2D Plot and (b) 3D Plot of the FNMRF system for $\alpha=0.8, \beta=1$, $\eta=0.9$ and initial condition $\left(M_{x}(0), M_{y}(0), M_{z}(0)\right)=(0,100,0)$ for $T_{\text {sim }}=0.1 \mathrm{~s}$.

Figure 8 represents the comparison between the approximate solution and the analytical solution of the fractional FNMRF system for $M_{x}(t), M_{y}(t)$ and $M_{z}(t)$, respectively $[23,24]$, for height steps $h=0.01$ and $h=0.001$.

We can see an excellent consistency of the solution and a similar result may also be detected for $M_{z}(t)$; see Table 1. From these observations, we conclude that the approximate solution well matches the analytical solution. The condition of stability for equation orders $\alpha=0.8, \beta=1, \eta=0.9$ of the solution is described in Figure 7 .

Figures 1-7, in 2D and 3D, illustrate the dynamics between $M_{x}(t), M_{y}(t)$, and $M_{z}(t)$. For both cases, magnetization of the entire trajectory is presented in 3D with the starting point $\left(M_{x}(0), M_{y}(0), M_{z}(0)\right)=(0,100,0)$ and the return to its equilibrium value of $M_{0}$.

Figure 8 shows the exact solution and the numerical solution obtained by applying the presented NIPCA method for (a) $h=0.01$ and (b) $h=0.001$, and initial condition $\left(M_{x}(0), M_{y}(0), M_{z}(0)\right)=(0,100,0)$ for $T_{\text {sim }}=1 \mathrm{~s}$. The maximum error in $\left(M_{x}, M_{y}, M_{z}\right)$ and simulation time $T_{\text {sim }}=0.1 \mathrm{~s}, 0.5 \mathrm{~s}$ and $1.0 \mathrm{~s}$ with time steps $h=0.1,0.01,0.001,0.05$, and 0.005 are listed in Table 1 . It is easily shown from Table 1 that the time step is directly proportional to the error. 


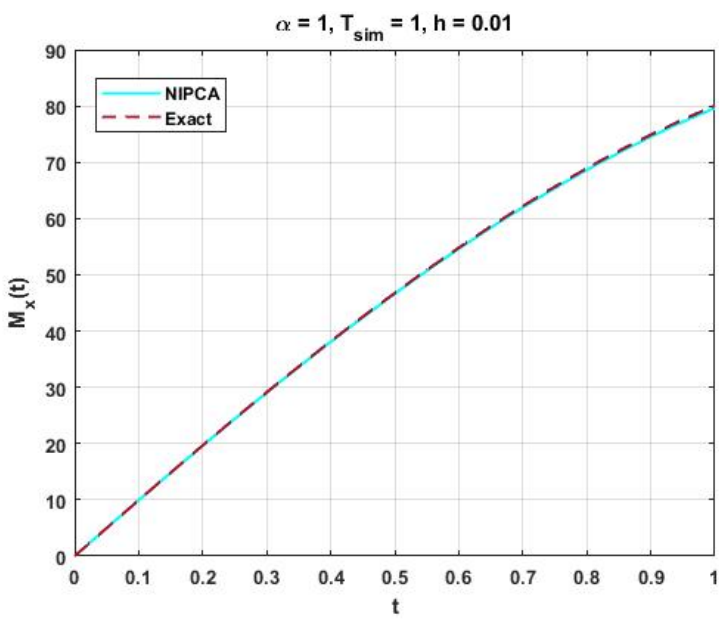

(a)

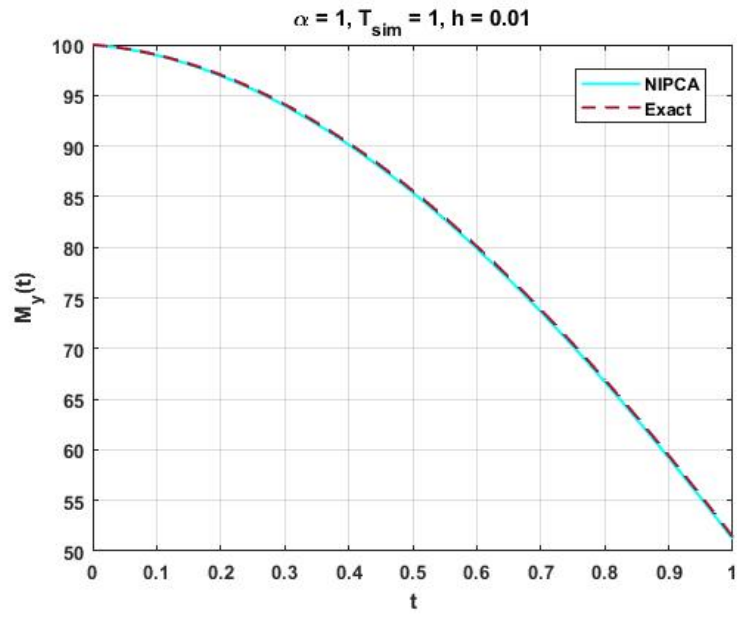

(c)

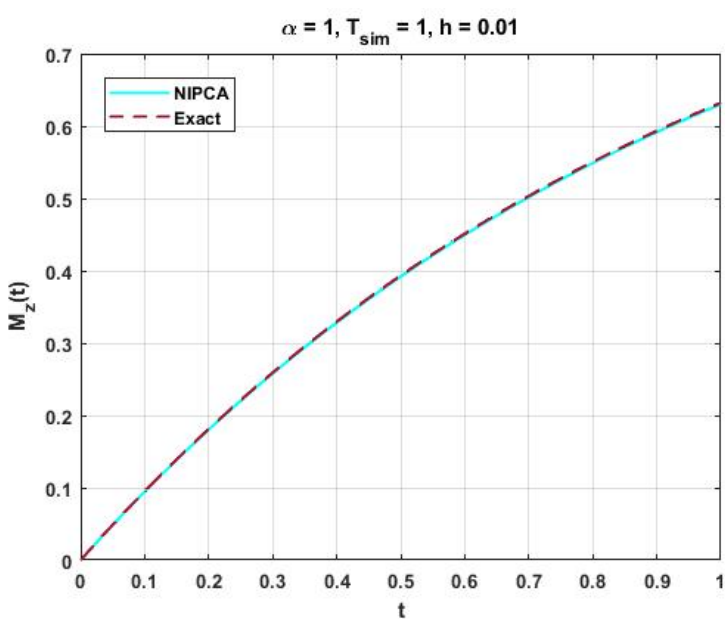

(e)

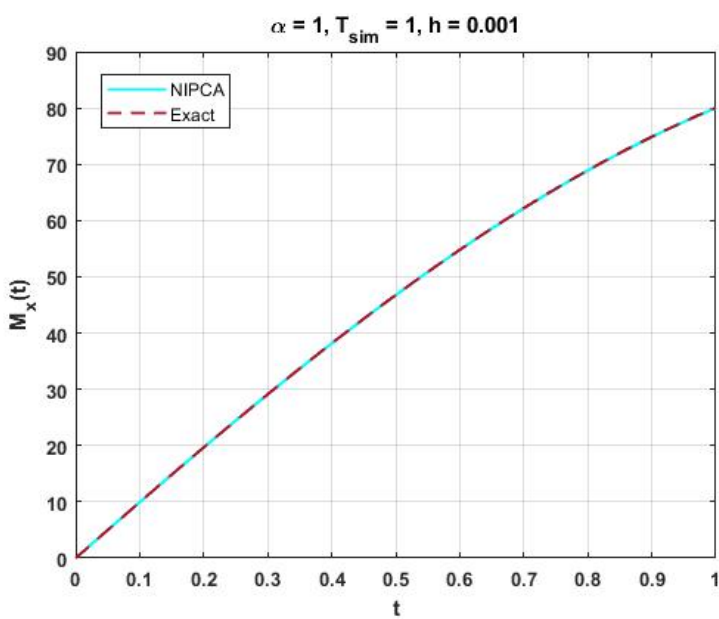

(b)

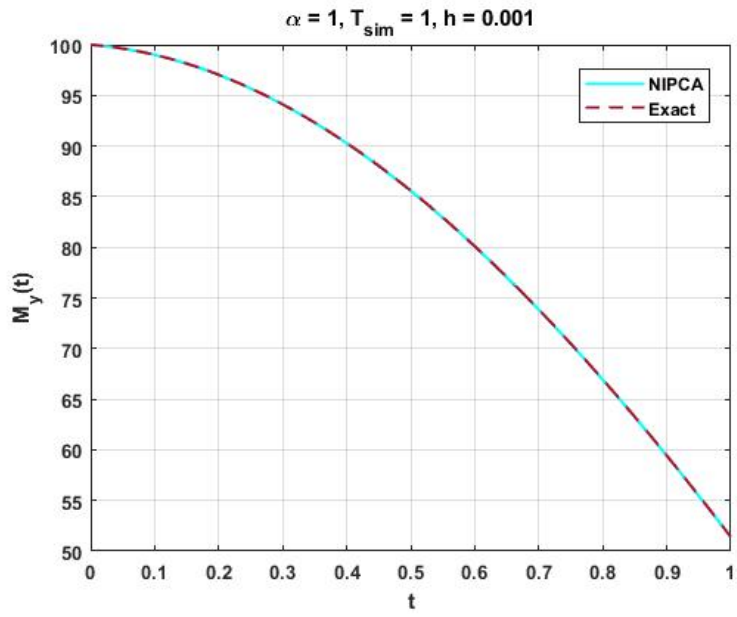

(d)

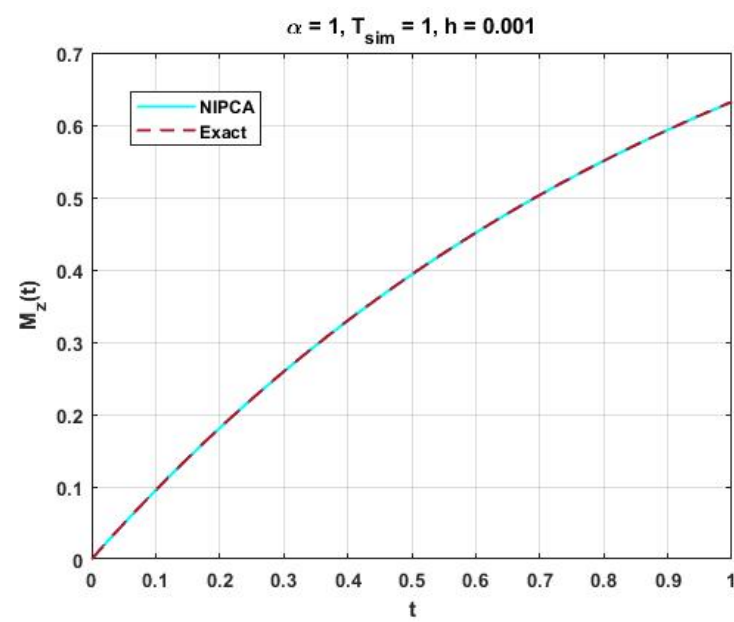

(f)

Figure 8. Comparison between the Exact solution and Analytical solution of the FNMRF system for $(\mathbf{a}) h=0.01(\mathbf{b}) \mathrm{h}=0.001(\mathbf{c}) \mathrm{h}=0.01(\mathbf{d}) \mathrm{h}=0.001(\mathbf{e}) \mathrm{h}=0.01(\mathbf{f}) \mathrm{h}=0.001$ and initial condition $\left(M_{x}(0), M_{y}(0), M_{z}(0)\right)=(0,100,0)$ for $T_{\text {sim }}=1 \mathrm{~s}$. 
Table 1. Average absolute error in the New Iterative Predictor-Corrector Algorithm (NIPCA).

\begin{tabular}{ccccccc}
\hline Simulation Time $T_{\text {sim }}$ & Components of $\boldsymbol{M}$ & $\boldsymbol{h}=\mathbf{0 . 1}$ & $\boldsymbol{h}=\mathbf{0 . 0 1}$ & $\boldsymbol{h}=\mathbf{0 . 0 0 1}$ & $\boldsymbol{h}=\mathbf{0 . 0 5}$ & $\boldsymbol{h}=\mathbf{0 . 0 0 5}$ \\
\hline \multirow{3}{*}{$T_{\text {sim }}=0.1$} & $M_{x}$ & $2.074 \times 10^{-2}$ & $2.776 \times 10^{-3}$ & $3.995 \times 10^{-4}$ & $6.210 \times 10^{-3}$ & $1.713 \times 10^{-3}$ \\
& $M_{y}$ & $2.485 \times 10^{-1}$ & $1.658 \times 10^{-2}$ & $2.369 \times 10^{-3}$ & $4.200 \times 10^{-2}$ & $1.009 \times 10^{-2}$ \\
& $M_{z}$ & $1.880 \times 10^{-5}$ & $1.910 \times 10^{-4}$ & $2.292 \times 10^{-5}$ & $3.963 \times 10^{-4}$ & $1.057 \times 10^{-4}$ \\
\hline \multirow{3}{*}{$T_{\text {sim }}=0.5$} & $M_{x}$ & $1.705 \times 10^{-1}$ & $4.708 \times 10^{-2}$ & $5.090 \times 10^{-3}$ & $1.573 \times 10^{-1}$ & $2.460 \times 10^{-2}$ \\
& $M_{y}$ & $5.674 \times 10^{-1}$ & $1.027 \times 10^{-2}$ & $1.102 \times 10^{-2}$ & $3.818 \times 10^{-1}$ & $5.339 \times 10^{-2}$ \\
& $M_{z}$ & $5.963 \times 10^{-3}$ & $8.645 \times 10^{-5}$ & $8.983 \times 10^{-5}$ & $3.657 \times 10^{-3}$ & $4.415 \times 10^{-4}$ \\
\hline \multirow{2}{*}{$T_{\text {sim }}=1.0$} & $M_{x}$ & $1.003 \times 10^{-1}$ & $1.562 \times 10^{-1}$ & $1.625 \times 10^{-2}$ & $6.476 \times 10^{-1}$ & $7.985 \times 10^{-2}$ \\
& $M_{y}$ & $1.257 \times 10^{-1}$ & $1.638 \times 10^{-1}$ & $1.694 \times 10^{-2}$ & $7.152 \times 10^{-1}$ & $8.342 \times 10^{-2}$ \\
& $M_{z}$ & $1.065 \times 10^{-2}$ & $1.292 \times 10^{-3}$ & $4.318 \times 10^{-4}$ & $5.922 \times 10^{-3}$ & $6.533 \times 10^{-4}$ \\
\hline
\end{tabular}

\section{Conclusions}

In this article, we obtained nonlinear fractional nuclear magnetic resonance flow equations (FNMRFEs) and the New Iterative Predictor-Corrector Algorithm (NIPCA) for their approximate solution. For NMR, the developed mathematical model allows the description and investigation of magnetization for spin dynamics (relaxation times $T_{1}^{\prime}$ and $T_{2}^{\prime}$ ) at the resonance frequency $\omega_{0}^{\prime}$ in a static magnetic field $B_{0}$. A New Iterative Predictor-Corrector Algorithm (NIPCA) was proposed to solve nonlinear fractional nuclear magnetic resonance flow equations. The time efficiency and high accuracy of the proposed technique are evident from the detailed error analysis of the FNMRFEs.

Author Contributions: M.S.: conceptualization, supervision, validation, formal analysis, writingoriginal draft preparation and writing - review and editing; U.A.: software, methodology, formal analysis, validation, data curation and writing-review and editing; M.K.: supervision, conceptualization, writing-original draft preparation and writing-review and editing; A.A.: writingreview and editing, visualization, investigation and resources; W.A.: methodology, formal analysis, investigation, funding acquisition, resources and visualization; H.Y.Z.: visualization, investigation, resources and funding acquisition. All authors have read and agreed to the published version of the manuscript.

Funding: This work received funding from King Khalid University, Ministry of Education. Princess Nourah bint Abdulrahman University, Saudi Arabia. Princess Nourah bint Abdulrahman University Researchers Supporting Project number (PNURSP2022R157), Princess Nourah bint Abdulrahman University, Riyadh, Saudi Arabia.

Institutional Review Board Statement: Not applicable.

Informed Consent Statement: Not applicable.

Data Availability Statement: Not applicable.

Acknowledgments: The authors express their appreciation to the Deanship of Scientific Research at King Khalid University for funding this work through the research groups program under grant number R.G.P.2/50/40. Also, the authors extend their appreciation to the Deputyship for Research \& Innovation, Ministry of Education, in Saudi Arabia, for funding this research work through the project number: (IFP-KKU-2020/9). Princess Nourah bint Abdulrahman University Researchers Supporting Project number (PNURSP2022R157), Princess Nourah bint Abdulrahman University, Riyadh, Saudi Arabia.

Conflicts of Interest: The authors declare no conflict of interest.

\section{References}

1. Singh, H.; Srivastava, H.M. Numerical Simulation for Fractional-Order Bloch Equation Arising in Nuclear Magnetic Resonance by Using the Jacobi Polynomials. Appl. Sci. 2020, 10, 2850. [CrossRef]

2. Johnston, E.R. Solution of the Bloch Equations including Relaxation. Concepts Magn. Reson. Part A 2020, 2020, 1-5. [CrossRef]

3. Ali, H.M. An efficient approximate-analytical method to solve time-fractional KdV and KdVB equations. Inf. Sci. Lett. 2020, 9, 189-198. 
4. Sultana, M.; Arshad, U.; Alam, N.; Bazighifan, O.; Askar, S.; Awrejcewicz, J. New Results of the Time-Space Fractional Derivatives of Kortewege-De Vries Equations via Novel Analytic Method. Symmetry 2021, 13, 2296. [CrossRef]

5. Zada, L.; Al-Hamami, M.; Nawaz, R.; Jehanzeb, S.; Morsy, A.; Abdel-Aty, A.; Nisar, K.S. A New Approach for Solving Fredholm Integro-Differential Equations. Inf. Sci. Lett. 2021, 10, 407-415.

6. Khater, M.M.A.; Attia, R.A.M.; Abdel-Aty, A.H. Computational analysis of a nonlinear fractional emerging telecommunication model with higher-order dispersive cubic-quintic. Inf. Sci. Lett. 2020, 9, 83-93.

7. Mohammadein, S.A.; Gad El-Rab, R.A.; Ali, M.S. The Simplest Analytical Solution of Navier-Stokes Equations. Inf. Sci. Lett. 2021, 10, 159-165.

8. Abragam, A. Principles of Nuclear Magnetism; Oxford University Press: New York, NY, USA, 2002.

9. Brown, R.W.; Cheng, Y.C.N.; Haacke, E.M.; Thompson, M.R.; Venkatesan, R. Magnetic Resonance Imaging: Physical Principles and Sequence Design; John Wiley and Sons: New York, NY, USA, 2014; ISBN 978-0-471-72085-0.

10. Singh, H. A new numerical algorithm for fractional model of Bloch equation in nuclear magnetic resonance. Alex. Eng. J. 2016, 55, 2863-2869. [CrossRef]

11. Caputo, M. Linear Models of Dissipation whose Q is almost Frequency Independent-II. Geophys. J. Int. 1967, 13, 529-539. [CrossRef]

12. Daftardar-Gejji, V.; Jafari, H. Adomian decomposition: A tool for solving a system of fractional differential equations. J. Math. Anal. Appl. 2005, 301, 508-518. [CrossRef]

13. Adomian, G. A review of decomposition method in applied mathematics. J. Math. Anal. Appl. 1988, 135, 501-544. [CrossRef]

14. Hemeda, A.A. Homotopy perturbation method for solving systems of nonlinear coupled equations. Appl. Math. Sci. 2012, 6, 4787-4800.

15. Bhalekar, S.; Daftardar-Gejji, V. Convergence of the new iterative method. Int. J. Differ. Equ. 2011, 2011, 1-10. [CrossRef]

16. Bhalekar, S.; Daftardar-Gejji, V. Solving a system of nonlinear functional equations using a revised new iterative method. Int. J. Math. Comput. Sci. 2012, 6, 968-972. [CrossRef]

17. Saeed, R.K.; Aziz, K.M. An iterative method with quartic convergence for solving nonlinear equations. Appl. Math. Comput. 2008, 202, 435-440. [CrossRef]

18. Daftardar-Gejji, V.; Jafari, H. An iterative method for solving nonlinear functional equations. J. Math. Anal. Appl. 2006, 316, 753-763. [CrossRef]

19. Khalid, M.; Sultana, M.; Arshad, U.; Shoaib, M. A Comparison between New Iterative Solutions of Nonlinear Oscillator Equation. Int. J. Comput. Appl. 2015, 128, 1-5. [CrossRef]

20. Khalid, M.; Sultana, M.; Zaidi, F.; Arshad, U. Solving Linear and Nonlinear Klein-Gordon Equations by New Perturbation Iteration Transform Method. TWMS J. App. Eng. Math. 2016, 6, 115-125.

21. Khalid, M.; Sultana, M.; Zaidi, F.; Arshad, U. An Effective Perturbation Iteration Algorithm for Solving Riccati Differential Equations. Int. J. Comput. Appl. 2015, 111, 0975-8887. [CrossRef]

22. Daftardar-Gejji, V.; Sukale, Y.; Bhalekar, S. A new predictor-corrector method for fractional differential equations. Appl. Math. Comput. 2014, 244, 158-182. [CrossRef]

23. Khalid, M.; Sultana, M.; Arshad, U. Procuring Analytical Solution of Nonlinear Nuclear Magnetic Resonance Model of Fraction Order. Sohag J. Math. 2017, 4, 69-73. [CrossRef]

24. Petráš, I. Modeling and numerical analysis of fractional-order Bloch equations. Comput. Math. Appl. 2011, 61, 341-356. [CrossRef] 